OPEN ACCESS

Edited by:

Rifat Hamoudi,

University of Sharjah,

United Arab Emirates

Reviewed by:

Julie Tabiasco,

Institut National de la Santé et de la

Recherche Médicale

(INSERM), France

Rania Harati,

University of Sharjah,

United Arab Emirates

*Correspondence:

Jean-François Rossi

jeanfrancoisrossi@me.com

Specialty section:

This article was submitted to

Cancer Immunity and

Immunotherapy,

a section of the journal

Frontiers in Immunology

Received: 17 August 2020

Accepted: 11 January 2021

Published: 23 February 2021

Citation:

Rossi J-F, Lu ZY, Massart C and Levon K (2021) Dynamic Immune/

Inflammation Precision Medicine: The

Good and the Bad Inflammation in

Infection and Cancer.

Front. Immunol. 12:595722.

doi: 10.3389/fimmu.2021.595722

\section{Dynamic Immune/Inflammation Precision Medicine: The Good and the Bad Inflammation in Infection and Cancer}

\author{
Jean-François Rossi ${ }^{1,2 *}$, Zhao Yang Lu ${ }^{3}$, Cesare Massart ${ }^{4}$ and Kalle Levon ${ }^{5}$ \\ 1 Hématologie-Immunothérapie, Institut du Cancer Avignon-Provence, Sainte Catherine, Avignon, France, 2 Faculté de \\ médecine Montpellier, Université de Montpellier, Montpellier, France, ${ }^{3}$ Unité de Thérapie Cellulaire, CHU Montpellier Saint-Eloi, \\ Montpellier, France, ${ }^{4}$ E-SANA, Orsay, France, ${ }^{5}$ New York University (NYU) Tandon School of Engineering, Six Metrotech Center, \\ Brooklyn, NY, United States
}

Normal or "good" inflammation process starts from a local cellular response against injury or any infectious agent, with the activation of neutrophils, macrophages, Langerhans cells, dendritic cells, and innate immune cells. Cytokines and chemokines are produced to amplify the local inflammatory process followed by the migration of immune cells to the regional lymph nodes where adaptive immune response is initiated. Systemic inflammation enhances the biological response to mobilize additional cells from central and peripheral immune/hematopoietic system. Local mechanisms to limit inflammation are initiated and lead to healing. During the normal inflammatory process, there is a balance between the production of inflammatory chemokines/cytokines such as Tumor Necrosis Factor (TNF)- $\alpha$, interleukin (IL)- 6 and IL-1 and the production of compounds that limit inflammation and have an immune suppressive effect, such as IL-10 and Transforming Factor (TGF) $\beta$. IL-6 and IL-6/soluble IL-6 Receptor (R) complex stimulate liver cells to produce inflammatory proteins, which represents the systemic inflammation response. The magnitude and the duration of the systemic inflammatory response are linked to the cause, under genetic and epigenetic control. Significant inflammation as seen in septic shock, in severe forms of infections or in certain active cancers, represents the "bad inflammation", correlated with a poor prognosis. In addition, the persistence of a chronic smoldering inflammation may lead to pathological situations which are observed in the majority of inflammatory, degenerative, dysmetabolic, or dysimmune diseases and cancer. Chronic smoldering inflammation is a cross between different pathological situations possibly linked. In addition, within the tumor microenvironment, inflammatory process results from different cellular mechanisms modulated by metabolic and vascular changes. On the contrary, a limited and balanced inflammation initiates the normal immune response, including the adaptive response which amplifies any immunotherapy, including vaccines. Immune checkpoint inhibitors and chimeric antigen receptor (CAR) T-cells are associated with cytokine release syndrome, a clinical risk leading to the use of anti-cytokine drugs. Nowadays, it is time to monitor the dynamic 


\section{inflammatory process for a better immune precision medicine in both infections} and cancer.

Keywords: inflammation, infection, cancer, precision medicine, immune therapy

\section{INTRODUCTION}

Inflammation is an essential and normal biological mechanism for response to an injury in humans. The first historical indications of inflammation, using herbology, was introduced in China by the mythic emperor Shennong, 5,000 years before. Hippocrates recommended extracts of willow bark to limit inflammation conducting to the salicilin identified in 1828 by Johannes Buchner and the chemical synthesis of acetylsalycilic acid by Charles Frédéric Gerhardt in 1853. The first description of the symptoms associated to inflammation, "rubor et tumor cum calore et dolore" was made in the first century by Aulus Cornelius Celsus. In the late $19^{\text {th }}$ century, inflammation associated to infection and the germ theory of disease was particularly introduced by Robert Koch and Louis Pasteur. In the same time, Rudolf Virchow observed infiltrated immune cells where cancer lesions appear in inflammatory tissue. In parallel, cellular aspects of the inflammation process were observed, including neutrophils and macrophages. In 1986, Dvorak defined tumors as "wounds that do not heal" and showed that carcinogenesis and inflammatory conditions have common developmental pathways (1). More recently, the cellular and molecular mechanisms of the local inflammation were identified with a local inflammatory response followed by a regional adaptive immune response and systemic inflammation to amplify the initial biological response.

Inflammation process is a protective response against foreign bodies or injuries, as observed in host tissues. It begins with enhanced vascular permeability of capillaries and leukocyte recruitment through endothelial activity. Normal controlled inflammation leads to healing and specific immune response through the activation of different mechanisms and particularly the antigen presentation with the activation and the maturation of antigen presenting cells. Amplified, uncontrolled, or prolonged inflammatory processes are associated with clinical symptoms and may lead to diseases including cancer. Systemic inflammation is associated to high proliferation rate and poor prognosis in cancer patients. The presence of inflammatory or immune cells within the tumoral microenvironment has been extensively studied particularly in the context of immune therapy aiming to predict clinical response to such treatment. On the contrary, the lack of a normal inflammatory response and/or an amplified immune suppression are also associated with pathological situations. In both sepsis and cancer, biological balance is essential, between a "good inflammatory response" that leads to a specific immune response, and a "bad inflammatory response" which is associated with an immune suppression leading to complications and disease progression. However, inflammatory process is changing over the time, reflecting the degree of the normal or abnormal response of the body. For all of these reasons, the dynamic evaluation of the inflammation process is mandatory in all diseases associated with inflammation. In this review, we discuss the biology of the normal and abnormal inflammatory response, trying to understand both the best analysis and thus, the best therapeutic targeting of such a dynamic by comparing inflammation during infection and cancer.

\section{CLINICAL OBSERVATIONS}

\section{"Good inflammation": The Beginning of a Normal Immune Response}

As observed in Figure 1, the favorable anticancer immune response observed in a particular patient having mantle cell lymphoma with poor prognosis was associated with a transient and limited inflammatory response. This observation illustrates a good inflammatory response which is the beginning of an adaptive immune response, both at cellular and humoral response levels. This controlled and transient inflammatory response is usually observed after vaccination and correlated to the reactogenicity, as described in hepatitis B and Salmonella typhi vaccines, and as shown also in Figure 1 (2-4). Different cytokines are associated with antigen presentation and maturation to cooperate with other immune cells (5). The time course and the magnitude of this inflammatory response

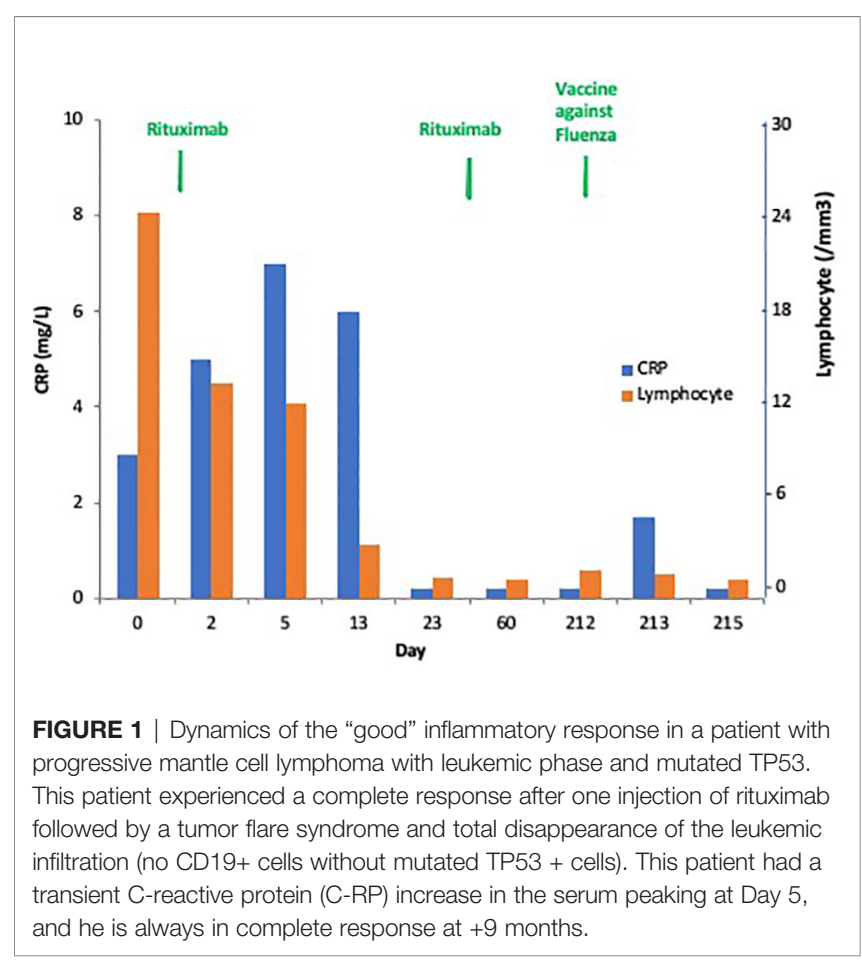


included serum interleukin (IL)-6 peaking at $12 \mathrm{~h}$ after an effective vaccination, ranging from 6 to $8 \mathrm{pg} / \mathrm{mL}$ and equally observed after each dose of vaccine. As expected, C-reactive protein (CRP) serum level peaks at day 1-2, ranging from 4 to 9 $\mathrm{mg} / \mathrm{L}$ and is correlated with the IL-6 peaks (2). Similarly, interferon- $\gamma$ and different serum levels of chemokines such as interferon $\gamma$ induced protein (IP)-10, macrophage inflammatory protein (MIP)-1 $\beta$ and monocyte chemoattractant protein (MCP)-2 were higher after the second dose of the most effective hepatitis B vaccine (2).

\section{"Bad Inflammation" Is Associated to Abnormal Immune Response}

A bad inflammation is an inflammatory response deregulated either in its duration or its amplitude. The cytokine storms observed during sepsis or after chimeric antigen receptor (CAR) $\mathrm{T}$-cell therapy are clinical situations associated with lifethreatening and represent an excessive inflammatory response (6-8). The presence of a smoldering chronic inflammation, described in the process of aging named inflammaging, as observed with congestive heart failure or chronic pulmonary disease, greatly reduces the specific immune response as demonstrated with varicella-zoster vaccine $(9,10)$. This lowlevel of persistent chronic inflammation has been observed to be associated with generalized atherosclerosis and used for cardiovascular risk stratification $(11,12)$.

\section{Inflammation and Cancer}

Inflammation is a well-known hallmark of cancer. A deregulated inflammatory process could be considered as the cause or consequence of cancer. Infectious diseases and chronic inflammation account for approximately $25 \%$ of cancercausing factors (13). Several infectious agents, including parasites, bacteria, and viruses have been demonstrated to be associated with different cancers through the production of reactive oxygen species (ROS) produced by inflammatory cells as well as epithelial cells, causing DNA damage in addition to epigenetic alterations.

Inflammation is associated with the risk of cancer, but also with the progression or metastatic risks, through different mechanisms such as chronic inflammation, tumor-associated inflammation, therapy-induced inflammation, or epigenetic conditions caused by environmental exposure or nutritional exposure (14). Paraneoplastic syndrome with B symptoms such as fever, sweats, weight-loss, and biological inflammatory symptoms, is correlated with a poor prognosis and advanced disease or metastatic risk $(15,16)$. In addition, excessive systemic inflammation is associated with immune suppression, thus amplifying the risk of secondary infection or the cancer progression (17).

In mice models, the creation of a local inflammation in the peritoneum leads to the appearance of a plasma cell tumor through the production of IL-6, a survival, and proliferation factor of myeloma cancer cells $(18,19)$. In plasma cell neoplasia, IL-6 is considered as a paracrine proliferation factor that may become an autocrine factor when the tumor proliferation became independent from the cancer micro-environment (20). IL-6 has been shown to be a survival and/or proliferation factor in certain cancers, such as kidney, ovarian, prostate, and certain types of Bcell lymphopathies, and can therefore be considered a therapeutic target (21). Generally, this very important inflammation is associated with a high proliferation rate of cancer cells which increases chemokine and cytokine production which attract even more immune cells into the cancer microenvironment and lead to tumor cell death and necrosis. The immune microenvironment of the tumor is changing under the pression of different elements, including hypoxia, angiogenesis, tumor mutations, and the production of different chemokines and cytokines in a permanent dialogue between cancer cells and immune surveillance supported by the immune effector cells (22). Hypoxia is associated with a metabolic change of immune cells, from mitochondrial respiration to glycolysis which turns the Tlymphocytes to T-regulator cells (T-reg) which share tolerogenic effects (23). In addition to T-regs, myeloid cells including myeloid derived suppressor cells (MDSC) and neutrophils have been associated with tumor progression and poor prognosis $(24,25)$. Immune suppression is initiated by the production of a panel of inflammatory cytokines including IL6, IL1, IL10, IL11, and transforming growth factor (TGF) $\beta$, mainly produced by macrophages (26). In addition to the changes observed within the cancer microenvironment, circulating MDSC concentrations, neutrophils, and neutrophil:lymphocyte ratio, are correlated with metastatic disease in different cancers such as melanoma, breast, and gastrointestinal cancers (27). The deregulation of different pathways implicated in cancer growth like the loss of tumor suppressors and/or activation of oncogenes influences the formation of an anti-inflammatory microenvironment. Due to the cytokine and chemokine changes, intracellular expression of transcription factors such as NF- $\mathrm{KB}$ or STAT3 is activated (28), a situation that is amplified in obesity (29).

\section{INFLAMMATION PROCESSES}

The onset of a normal acute inflammatory response requires triggering factors, rapidly followed by a repair phase reflecting the transient nature of this process (Figure 2). The inflammatory process has been particularly studied in the context of bacterial infection. However, there is a common inflammatory response to any injury, with inducers, sensors, mediators, and effectors (30).

\section{Inducers}

Inducers can be exogenous or endogenous. Exogenous inducers are infectious or non-infectious stimuli and are classified as pathogen-associated molecular patterns (PAMPs) and damageassociated molecular patterns (DAMP) or "alarmins" that are released from damaged cells of the host (31). PAMPs included nucleic acids particularly from viruses or bacteria, proteins such as pilin and flagellin from bacteria, cell wall lipids such as lipopolysaccharide (LPS), lipoteichoic acid from bacteria, or carbohydrates (mannan or glucans) from fungi or bacteria (32). DAMPs are stress-induced proteins such as heat shock 


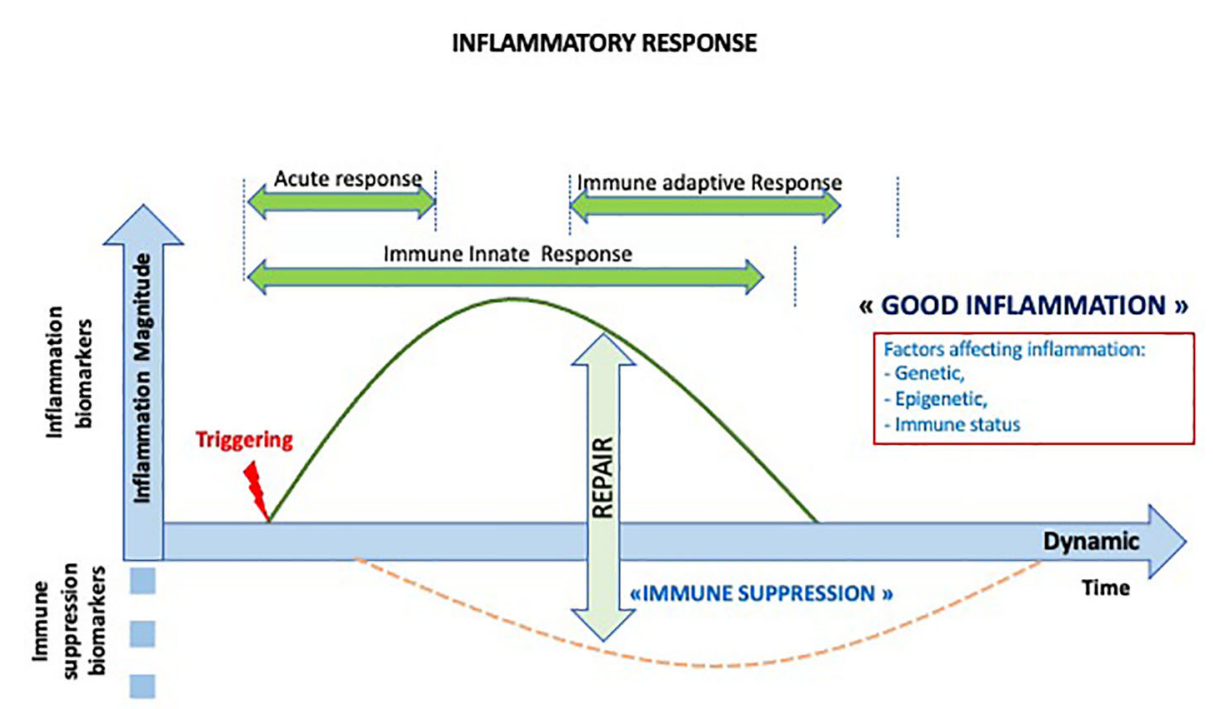

FIGURE 2 | Dynamics of the normal inflammatory response. The inflammatory response is a transient response followed by transient immune suppression and repair period and the initiation of the adaptive immune response.

proteins (HSP), crystals proteoglycans, mitochondrial components or nuclear proteins such as high mobility group box 1 (HMGB1), several members of the S100 calcium-binding protein family (S100A8, S100A9 and S100A12), histones, and other molecules released when cells are damaged (33). All of these endogenous inducers engage different sensors which are specialized cell-associated recognition molecules of the innate immune system that are activated by the inducers and trigger the production of mediators.

\section{Sensors}

The different endogenous inducers of inflammation obtained after necrotic cell death have also different sensors, such as advanced glycation end-product-specific receptor (RAGE) for HMGB1 and S100A12, purinoceptors (including P2X) for ATP binding resulting in $\mathrm{K}^{+}$ion efflux and cooperate with the NACHT, LRR, and PYD domains containing protein (NALP)3 inflammasome. Such inducers may have crossed or specific activities, like ATP which also activates nociceptors reporting tissue injury to the nervous system, or receptor for RAGE that cooperates with Toll-like receptors (TLRs) (30). There are also soluble of PAMPs, including, pentraxins such as CRP, collectins, ficolins, or complement (C3b) molecules $(34,35)$. Sensors are expressed by phagocytes, primarily macrophages and neutrophils, dendritic cells (DC), epithelial cells at the barrier of the body, endothelial cells, mast cells, and other types of cells within tissue. Some of these pattern recognition receptors are cell associated including TLRs (1-9), nucleotide oligomerization domain receptors (NOD)-like receptors (NLRs, NOD1-2, inflammasomes), retinoic acid-inducible gene RIG-like receptors (RLRs) (MDA-5), cytosolic DNA sensors (CDSs), Ctype lectin-like receptors (CLRs), including mannose receptor, DC-sign, Dectin-1 and 2, scavenger receptors (CD36), N-Formyl met-leu-phe receptors (34).

\section{Mediators}

Inducers of inflammation trigger the production of several inflammatory mediators. Many of these mediators are derived from plasma proteins or secreted by cells, mainly resident mast cells, platelets, basophils or macrophages. These mediators have effects on the vasculature and the recruitment of leukocytes and are classified as vasoactive amines, vasoactive peptides, fragment of complement components, lipid mediators, cytokines, chemokines, and proteolytic enzymes (30). Lipid mediators derived from phosphatidylcholine, included eicosanoids and platelet activating factors. In addition to the promotion of inflammation, mediators also initiated tissue repair after injury. Inflammasomes are cytosolic protein platform assembled in response to danger signals. Inflammasome complexes contain a sensor protein, an adaptor protein and a zymogen, procaspase- 1 that is activated into an active enzyme, caspase-1. Activated caspase- 1 subsequently activates pro-inflammatory cytokines IL$1 \beta$ and IL-18, and through the activation of gasdermin D, it induces pyroptosis, a highly pyrogenic inflammatory form of death $(34,36,37)$.

After DAMP or PAMP activation, neutrophils are activated directly or indirectly through the production of inflammatory mediators such as (C-X-C motif) ligand (CXCL)-1 and CXCL-2 which binds to and activate G-protein-coupled receptors on neutrophils (38).

\section{Effectors}

Effectors are all the cells present or called to the initial site of the injury. They include neutrophils, natural killer (NK) cells, monocytes/macrophages and T- and B lymphocytes. This local response is prolonged and amplified by a regional and a systemic inflammatory/immune response as shown in Figure 3. 


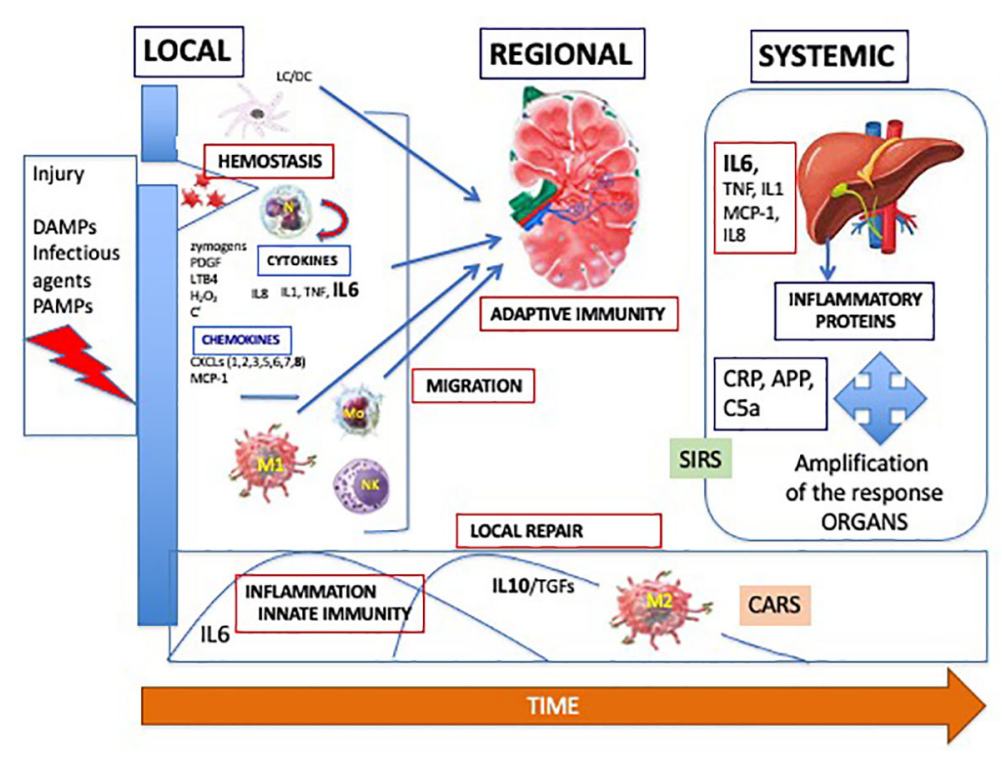

FIGURE 3 | Dynamics of the adapted inflammatory/immune response, with a local, regional in the lymph node and systemic processes. DAMPs, damageassociated molecular patterns; PAMPS, pathogen-associated molecular patterns; PDGF, platelet activating factor; LTB4, leukotriene B4; C, complement; IL, interleukin; TNF, tumor necrosis factor; CXCL, chemokine C-X-C- motif ligand; MCP-1, monocyte chemoattractant protein; M, macrophage; NK, natural killer; Mo, monocyte; TGF, transforming growth factor; CARS, compensatory anti-inflammatory response syndrome; SIRS, systemic inflammatory response syndrome; CRP, C-reactive protein; APP, amyloid protein P.

Neutrophils arrive at sites of damage or infection within minutes after migration along chemotactic agents. Intravascular migration of neutrophils starts by "rolling" on the endothelium of blood vessels which is mediated by selectins and followed by chemokine activation through a conformational change of the $G$ protein coupled receptor leading to activation of neutrophil integrins such as very late antigen (VLA)-4 (CD49D/CD29), macrophage-1 antigen (MAC$1, \mathrm{CD} 11 \mathrm{~b} / \mathrm{CD} 18)$, and lymphocyte function-associated antigen 1 (LFA-1, CD11a/CD18) (39). This integrin activation favors the cell adhesion to endothelium through the Immunoglobulin (Ig)superfamily cell adhesion molecules (ICAMs). They shed microvesicles that include chemotactic signals such as leukotrienes from arachidonic acid from lipid membranes after inflammasome activation (40). Neutrophils have a relatively short life, dying via apoptosis particularly in anti-bacterial activity that include phagocytosis and the production of ROS, proteases, and neutrophil extracellular traps (NETs) (41). NET is a network associating DNA coated with histones, elastase, myeloperoxidase (MPO), and cathepsin G, initially observed in bacterial cell killing and leading to cell death and named lytic NET $(42,43)$. In addition, it was demonstrated that NET formation can occur independent of cell lysis and subsequent cell death in non-infectious stimuli. In such a non-lytic NET release, this mechanism appears more rapidly in 5 to $60 \mathrm{~min}$ as compared to 3 to $5 \mathrm{~h}$ for the lytic NET release. This non-lytic NET release is mitochondrial and not nuclear, lacking histones (44). Recently, it was demonstrated that it is not all neutrophils that release NETs, but more particularly the low-density neutrophils, generally CD177 negative and expressing olfactomedin 4 (OLFM4) and which secrete this molecule after the release of NET (45). A recent study has shown that patients with a high percentage of OLFM4+ neutrophils are associated with high risk of septic shock and organ failure (46). In addition, OLFM4 forms complexes with a number of binding molecules included in major signaling pathways such as nuclear factor kappa B (NF- $\mathrm{KB}$ ) and wingless Int-1 (Wnt), that play main roles in carcinogenesis and inflammation. However, neutrophils are cells difficult to analyze, due to their short life span, their changes after ex vivo manipulations. Recent advances, using automated flow cytometers, have permitted to better analyze neutrophil subpopulations based on their size, nuclei aspect, and the presence of different surface biomarkers including CD62L, C5aR, CD11b, Fc $\gamma$ RII, associated with inflammatory/immune activation as well as immune suppression (47). MPO is mainly produced by neutrophils and belongs to the family of hemecontaining peroxidases. Its activity involves the production of ROS and is released into extracellular fluid after oxidative stress and inflammatory responses (48). Thus, a controlled MPO release at the site of infection is crucial, and abnormal release or function is associated to different diseases expressing chronic inflammation. Cell-cell interactions between neutrophils, endothelial cells, monocytes, and lymphocytes are essential, with the expression of different molecules interaction including integrins.

Neutrophils and monocytes/macrophages arise from common precursors, and co-express similar antigens and produce similar effector molecules including chemokines, cytokines, oxidants (49). However, they have different activities in the inflammation process, particularly in defense against 
pathogens. Neutrophils are the first cells present in injured tissue, having higher microbiocidal activity, whereas monocytes/ macrophages are recruited later on, and they participate to the initiation of adaptive immune response through digestion and antigen presentation. Monocytes are heterogeneous cells that circulate in peripheral blood, representing a central cell family in the context of innate immunity and inflammation. In humans, three subpopulations of monocytes have been described, including CD14++CD16- classical, intermediate CD14+CD16+, nonclassical CD14-CD16++ monocytes (50). Tissue-resident macrophages have major functions in resolution of inflammation and tissue repair (51). Neutrophils are programmed to quickly die to prevent excessive inflammation. Macrophages act to prolong their survival by producing growth factors such as granulocyte-macrophage colony-stimulating factor (GM-CSF), G-CSF, and tumor necrosis factor alpha (TNF- $\alpha$ ) (52). Macrophages are able to acquire either the "M1" pro-inflammatory phenotype or the "M2" antiinflammatory phenotype (53). M1 macrophages express inducible nitric oxide synthetase and CD40. They produce cytokines such as IL- 6 and TNF- $\alpha$ and are particularly active in case of infection (49, 54). M2 macrophages express arginase I and CD206 and produce TGF- $\beta$ and IL-10 to start tissue repair. Neutrophil heterogeneity and plasticity have been recognized and these cells may differentiate into different subsets that interact and modulate macrophage activity. The activity of neutrophils and the polarization of monocytes/macrophages are associated to metabolic changes due to the energy required and REDOX regulation. Neutrophils are more dependent on glycolysis to provide rapid energy necessary to ROS production. Monocytes first use mitochondrial oxidative phosphorylation and glycolysis depending the phase of the immune response, primarily inflammation and then resolution and tissue repair activation (40).

\section{Inflammation Homeostasy Regulation and Dynamics}

The regulation of inflammation takes place in a sequence of initiation, amplification and control of the inflammatory process with a view to repair. The mechanisms present during these different phases bring into play the mediators and cells described previously. The homeostatic regulation maintains the levels of glucose and oxygen concentrations at acceptable range depending the level of inflammation which is extreme in the context of infection and injury, and the lack of any tissue malfunction.

The inflammatory response is a dynamic controlled response. Thus, the analysis of the pathological inflammatory response cannot be conceived of apart from a dynamic study relating to its amplitude and duration. As shown in Figure 4, there are several pathological situations, including the cytokine storm observed during infections, inflammatory cancers generally associated with a poor prognosis or immunoresistance, and persistent chronic inflammation, a risk factor for disease.

The pro-inflammatory response is generally self-limited, even in the absence of effective therapy. However, in some patients who develop sepsis, the response is hyper-amplified, associated to a "systemic inflammatory response syndrome" (SIRS) and leads to a compensatory down regulation of the immune system, named "compensatory anti-inflammatory response syndrome" (CARS) (55).

This dynamic aspect has been observed for CRP on a longer period mainly in the context of infection. The slow decline in CRP after inflammatory process, as well as the peak of CRP serum level are associated with longer survival in older hospitalized patients on a 6-month period (56). However, this dynamic CRP evaluation does not take in account of the early

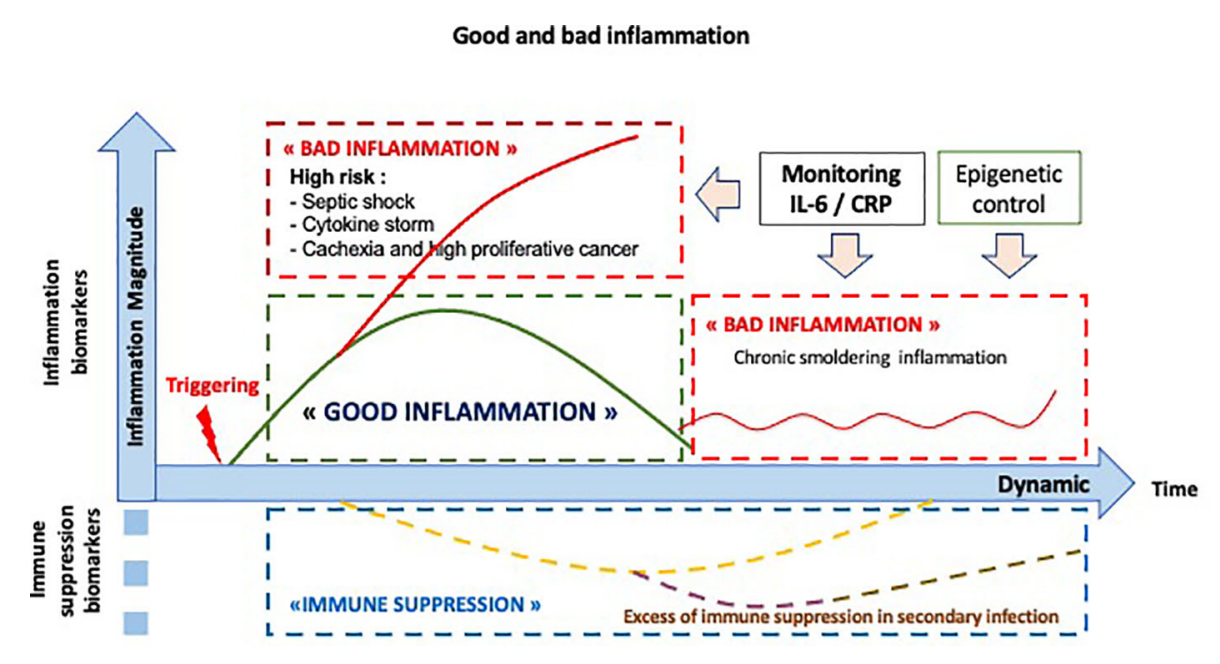

FIGURE 4 | Dynamics: the normal and abnormal inflammatory response. The normal inflammatory/immune response is noted in green. The abnormal inflammatory/ immune responses are noted in red, including a high level of inflammation, as observed in septic shock or in cytokine storm, a chronic smoldering inflammation or an excess of immune suppression, named CARS (compensatory anti-inflammatory response syndrome) in infection process. 
and rapid changes in inflammatory process, the delayed response and the half-life of the molecule, thus reflecting a more complex physiological process.

\section{BIOMARKERS OF INFLAMMATION}

Biomarkers of the inflammatory response are the mediators and effectors of such a process. Usual cites of choice for these biomarkers have centered on their significance with respect to specific diagnostic settings including infection, cancer, trauma, and other pathological situations. The dynamic aspect introduces a three-dimensional vision, time and therefore its duration, amplitude, and speed of its constitution, making it possible to locate the normal or abnormal nature of this inflammatory response as shown in Figure 4.

\section{Effectors}

Neutrophil/monocyte lineage and innate cells are early effector cells particularly after an acute injury, such as infection. Analysis of circulating neutrophils, particularly those expressing CD16bright CD62Ldim and described as precursors of MSDC, as well as subpopulations of monocytes were evaluated by flow cytometry for the early detection of infections or after immune suppression (39). Monocyte Human Leucocyte Antigen (HLA)DR expression decreases rapidly in correlation to the severity and outcome of the septic shock (57). Dynamic changes of monocyte phenotypes have been demonstrated to be associated with severity of the COVID-19 disease, particularly HLA-DR ${ }^{\text {lo }}$ CD $163^{\text {hi }}$ monocytes were present mainly early in severe disease, while HLA-DR ${ }^{\text {lo }}$ S100A ${ }^{\text {hi }}$ monocytes dominated the late phase of disease (58). Recent technological advances have made it possible to better understand the phenomenon of trogocytosis, an essential mechanism of intercellular communication, particularly observed with immune cells in cancer as well as in infection $(59,60)$.

\section{Early Mediators}

\section{Cell Surface Molecules Shedding}

Upon leukocyte activation with diverse stimuli, adhesion molecules are rapidly shed from cell surface by proteolytic cleavage. Three of them belonging to the selectin family (Eselectin, L-selectin and P-selectin) and two belonging to the immunoglobulin domain superfamily cell adhesion molecules (intercellular adhesion molecule (ICAM)-1, vascular cell adhesion protein (VCAM)-1), were particularly associated with sepsis (61). The soluble form of cell surface CD14 molecule or presepsin, the receptor of lipopolysaccharide (LPS) is also considered as a biomarker for early infection and its severity and prognosis (62).

\section{Chemokines and Cytokines}

Among the different cytokines and chemokines produced at the initial phase of the inflammatory process, IL-8, and particularly IL- 6 and TNF- $\alpha$ have been extensively studied in inflammatory diseases and infections. They represent the main inflammatory cytokines released in the context of inflammation regardless its cause. IL-6 is a pleiotropic cytokine, with a four-helix bundle structure, having 212 amino acids with a signal peptide of 27 amino acids. Its molecular weight ranges between 21 and $28 \mathrm{kDa}$, depending on the amount of post-translational modifications, such as glycosylation and phosphorylation (63). IL-6 is produced by different types of cells, mainly fibroblasts, keratinocytes, monocytes, and macrophages, particularly in early phase of infectious inflammation after stimulation of Toll-like receptors.

In addition to IL-6, monocyte chemotactic protein (MCP)-3 and interferon gamma induced protein (IGP)-10 are early biomarkers of inflammation and have been observed to be excellent predictors for the progression of COVID-19 (64). In bronchoalveolar lavages from patients with severe and critical COVID-19 infection, IL-6 concentration may reach $10,000 \mathrm{pg} / \mathrm{mL}$, 10 to 100 -fold higher than that observed in the circulation reflected by the serum CRP concentration. This huge local production of cytokines is probably dependent on both the viral load and the magnitude of the macrophage and neutrophil activation (65).

A normal inflammatory response is followed by the return to a normal situation with immune suppression and repair. Different effectors and mediators are implicated in such a balanced response. Among them, the IL-10 family cytokines are essential to restore the host to an immune quiescent status and tissue homeostasis after inflammatory process. In infection, high levels of serum IL-10 are correlated with mortality in several studies, probably more through the immune suppression status and the risk of secondary infection or in persistent chronic infection particularly observed in mycobacterial or parasite infections $(66,67)$. In cancer, IL-10 was reported to have paradoxical effects in that both tumor-promoting and tumor-suppressive effects have been observed $(68,69)$. Thus, dynamic monitoring of serum follow-up of IL-10 in addition to inflammatory proteins and cytokines can provide additional information with regard to the inflammation-immunosuppression balance and its associated risks.

\section{Secondary Inflammatory Molecules}

In physiological conditions, IL-6 modulates the transcription of several hepatic genes, particularly $\operatorname{CRP}(70,71)$ and other acutephase proteins like serum amyloid A (SAA), $\alpha 1$-antichymoptrypsin, fibrinogen, haptoglobin, and hepcidin that is implicated in inflammatory anemia through the blockade of ferropontin 1, an iron transporter (72-74). On the opposite, IL-6 reduces the level of albumin, transferrin, fibronectin, and cytochrome P450, and more recently it was demonstrated that CYP3A4 mRNA expression was most reduced by IL-6 followed by CYP2C9 and CYP2C19 (75).

CRP has been extensively studied as a biomarker of both acute and chronic inflammation. Elevated CRP/IL6 levels in the serum are a hallmark of inflammatory and aggressive cancers, particularly associated with lung, colon, and ovarian cancers (76, 77). In addition, in ovarian cancer, a meta-analysis suggests that high CRP levels superior to $10 \mathrm{mg} / \mathrm{L}$, rather than circulating proinflammatory cytokines might contribute to the etiology of ovarian cancer (77). As CRP has a longer half-life, its dosage may represent an easiest dosage than IL-6 measurement for large 
cohort analysis particularly for risk evaluation (78). However, as CRP has no specificity, the definition of chronic inflammation necessitates a dynamic view using highly sensitive dosage, outside the context of an acute inflammation. In a recent meta-analysis pooling 83,995 participants from 14 studies, elevated CRP using a highly sensitive test has been shown as an independent predictive biomarker of mortality both for all causes with pooled RR at 1.75 (95 CI 1.55-198) and for cancer mortality, particularly in men, with pooled RR at 1.25 (CI 1.13138) (79). In the Copenhagen General Population Study with approximately 63,500 individuals, those with a high baseline CRP ( $>3 \mathrm{mg} / \mathrm{L}$ ) had an $80 \%$ greater risk of early death compared with those with low CRP levels $(<1 \mathrm{mg} / \mathrm{L})(80)$. Approximately $70 \%$ to $90 \%$ of colorectal cancer (CRC) arise from adenomatous polyps and it develops trough a gradual accumulation of genetic and epigenetic changes, increasing with age and chronic inflammation associated with obesity, inflammatory bowel diseases, and dysibiosis. CRP has been associated with a higher risk of CRC in some studies, but this correlation could have some biais, or high levels of CRP just represent the resultant of different causes leading to a chronic inflammation (78).

Questions also arise for the different biomarkers as cause or consequence of a cancer status (81). Different gene polymorphisms of CRP and inflammatory cytokines or biological mechanisms implicated in inflammatory process have been explored with heterogeneous concluding data, depending the type of gene polymorphism and the type of cancer, mainly in colorectal cancer (81). In the Rotterdam study, baseline CRP levels are associated to chronic inflammation preceding lung cancer, even after subtracting a 5-year latent period, as well as a single nucleotide polymorphism of CRP variation of CRP gene (82).

In the context of infection, other biomarkers have been extensively studied, including pancreatic stone protein/ regenerating protein (PSP/reg) and procalcitonin (PCT). PSP/reg is a lectin-binding protein that was firstly described in pancreas and was shown to activate polymorphonuclear cells. It is considered as an acute-phase protein regulated by cytokines produced in the damaged tissues. Recently elevated serum levels of PSP/reg have been correlated with the severity of infection, particularly in adults and newborns (83). PCT is a member of the calcitonin gene-related peptides and the precursor of the hormone calcitonin. The produced PCT is stored in the Golgi apparatus, explaining the low concentrations observed in the blood circulation. Many cytokines except interferon $\gamma$ contributed to the up-regulation of CALC-1 observed in all cells of the organism, resulting in the higher levels in bacterial infections. PCT has a halflife of 22-29 $\mathrm{h}$ and the secretion in bacterial infections starts to rise $4 \mathrm{~h}$ after the onset of infection, peaking at 12-24 h, earlier than CRP which peaks at 2-3 days with a half-life of 12-24 h (84). The intra- and inter-individual variation of PCT concentration in the peripheral blood limits its interpretation as a single dosage, thus needing serial measurements for a dynamic evaluation (85). Several prohormones, such as adrenomedullin, atrial natriuretic peptide, and arginine vasopressin have been associated with pneumonia (86). Triggering receptor expressed on myeloid cells (TREM)-1) is a glycoprotein member of the Ig family which is upregulated during inflammation linked to infection as well as in non-infectious inflammatory conditions (87). TREM-1 is not detectable in healthy individuals but only measured in body fluids in response to infection (55).

However, CRP is the strongest and easiest biomarker, largely diffused and used but also unspecific in lower dosages (under 90 $\mathrm{mg} / \mathrm{L}$ ). Heterogeneity of the quality of the results is partly due to the technique used in the different studies, mainly plasma (or serum, sometimes), sample collection variable from $4 \mathrm{~h}$ to $48 \mathrm{~h}$, variation of the frozen materials, all of these technical points impacting on the IL-6 dosage with short-half life and rapid degradation particularly in the serum. The kinetics of the PCT are between that of IL- 6 and that of CRP, mainly associated with bacterial infection. A decrease of PCT has been associated with antibiotic prescription, except for high-are risk patients, such as febrile neutropenia.

Thus, there is no ideal biomarker in acute inflammation/ infection. Consequently, there is a need for several biomarkers, including biomarkers for cellular activity, dynamic evaluation and particularly the analysis of the balance between the proinflammatory and the anti-inflammatory phases. Systemic inflammation is probably deleterious depending its magnitude and/or its persistence as observed in chronic inflammation.

During septic shock and particularly COVID-19 infection, the cytokine storm can cause clinical deterioration at high risk of death, in particular due to the rapidity of its constitution, as measured by IL-6/CRP (88). The dynamic measurement of IL6/ CRP is crucial to identify such a critical period, aiming to rapidly control such a clinical situation, generally by administering antiIL6 or anti-IL6R monoclonal antibodies. In addition, during anti-IL6 therapy, the measurement of CRP represents the main biomarker for defining the efficacy and in particular through the regression curve of this biomarker. Biological efficacy of such therapies is obtained when the circulating CRP is totally abrogated meaning that the bioactive IL-6 is neutralized (89). The optimal CRP regression curve can be determined, knowing its half-life which is above $19 \mathrm{~h}$, and we develop a mathematical model for optimizing such therapy (submitted for publication).

We have established a highly effective protocol and analysis for extended gate FET electronic measurement of the ELISA test using same sandwich-assay approach (90). This methodology was recently developed to measure the levels of CRP in serum from a drop blood (E-Sana SAS, Paris, France). The detection range was optimized for $10 \mu \mathrm{g} / \mathrm{ml}$ cutoff and detection limit observed at $10 \mathrm{ng} / \mathrm{ml}$ (submitted to publication). This new Pointof Care rapid immunoassay application will be multiplexed with other inflammatory proteins such as IL-6, opening the way for dynamic analysis of the inflammatory response as well as to explore new zones of the normal fluctuating inflammation.

\section{INFLAMMATORY/IMMUNE PRECISION THERAPY}

Serum CRP and SAA levels have demonstrated to be correlated with circulating IL- 6 concentration, thus representing also the best surrogate biomarkers for monitoring patients receiving anti-IL-6 


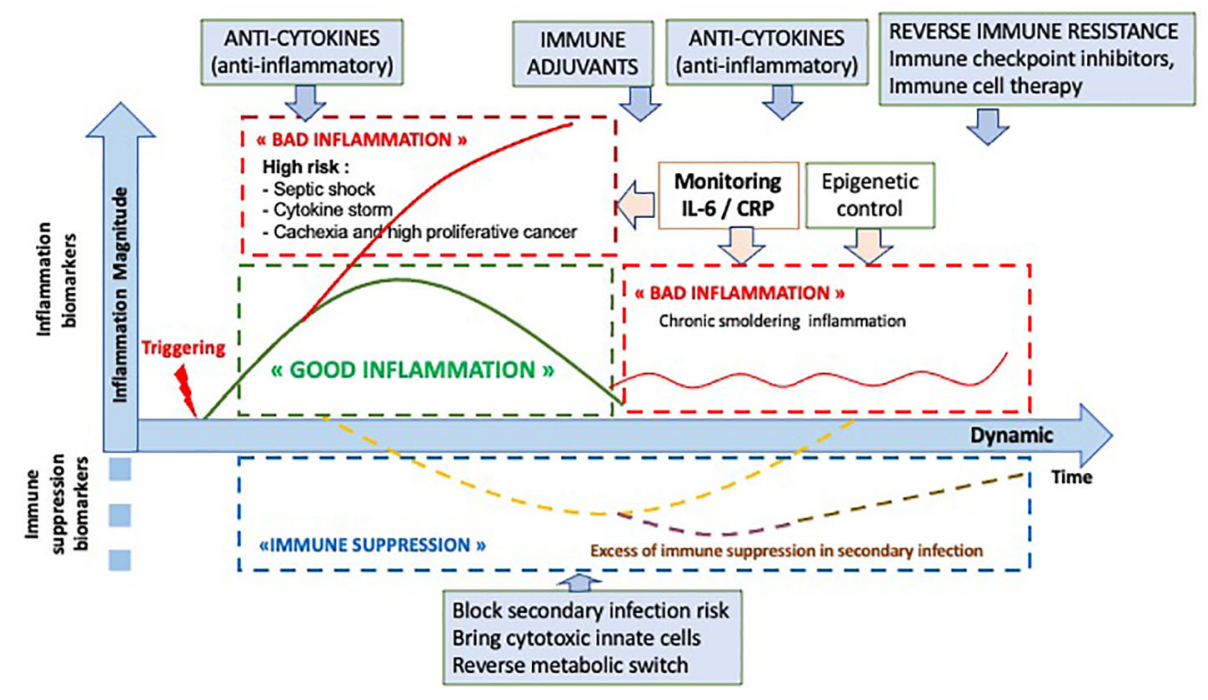

FIGURE 5 | Optional therapeutic interventions depending the dynamic analysis of the inflammatory and immune response.

treatments (21). Dynamic analysis of the inflammatory biomarkers may contribute to a more personalized immune medicine, monitoring both the risks associated to vaccine and the therapies needed, aiming to reduce inflammation by using anti-cytokine targeting IL-1, TNFo, or IL-6, or to amplify an immune response by using immune adjuvants (91, 92). IL- 6 was shown to play a major role in the maturation of DC, maintaining them at an immature status which is associated with tolerance (93). During the early steps of an infection process, the inflammatory response initiates and amplifies the immune response including DC maturation. At a later stage, newly formed immature DCs are locally induced by an immunological scare left-over by inflammation to amplify tolerance (94).

Under therapy such as immune checkpoint inhibitors, anticancer vaccines or combined therapies, systemic inflammation status at baseline predicts the outcome of the patients (95). However, it is difficult to define the prognostic value of IL-6/CRP in these different studies, due to the variability of the methodology used, either below the median or with different subgroups of values (85-87). Finally, it is clear that the lower CRP levels were associated with a better prognosis (96-98). IL-6 mediated STAT3 activation in the tumor microenvironment inhibits functional maturation of DC to activate effector T-lymphocytes, blocking the anticancer immunity leading to a therapeutic blockade of the IL-6 complex (98). IL-6 signaling has been shown to have both pro- and antiinflammatory activity, first directed to macrophages and T-and Blymphocytes differentiation and then promoting the resolution of inflammation (99). The usual cis-signaling is restricted to cells having the IL-6R $\alpha$, including liver cells and hemopoietic cells. IL6 trans-signaling represents the circulating complex IL-6/IL-6R $\alpha$ binding to the ubiquitous membrane gp130 signal transducer, and it has been shown to contribute to anti-tumor adaptive immunity by guiding lymphocyte trafficking to lymph nodes and tumors. So, it appears important to modulate more precisely the inflammation process, particularly in the context of immunotherapy, and not only to limit the toxicity. The use of anti-IL-6 or anti-IL-6R therapies necessitates reviewing the use of these drugs with the search for an immune modulation through precise and dynamic biological monitoring, including the soluble forms of IL-6 receptors as well as the different conformational changes of CRP, monomeric, and pentameric (100).

In addition, anticancer vaccine administration is followed by a significant but transient increase of CRP/IL-6 peaking at $24 \mathrm{~h}$ (101). Regarding the origin of these circulating proteins, it has been demonstrated that the serum levels of IL- 6 originate, at least in part, from the vaccine site $(101,102)$. Correlation between inflammation level and clinical outcome under immune therapies are controversial with both positive and negative correlation $(102,103)$. These contradictory data are due to the lack dynamic analysis of inflammation.

In conclusion, the inflammatory response is a normal mechanism to initiate the immune response. Its prognostic impact is known, in particular in the context of an excessive inflammatory response such as cytokine storm, as observed during infections or after CAR T-cell therapy. However, the impact of a good inflammatory response requires dynamic monitoring to observe its time course, amplitude and duration. This dynamic vision will thus make possible to resolve interpretation contradictions, opening the way to a new immune precision medicine both in cancer and infections as shown on Figure 5.

\section{AUTHOR CONTRIBUTIONS}

J-FR and ZL made the concept and wrote the article. KL and CM discussed the article and participated to the discussion around technical development. All authors contributed to the article and approved the submitted version. 


\section{REFERENCES}

1. Dvorak HF. Tumors: wounds that do not heal. Similarities between tumor stroma generation and wound healing. N Engl J Med (1986) 315:1650-9. doi: 10.1056/NEJM198612253152606

2. Burny W, Marchant A, Hervé C, Callegaro A, Caubet M, Fissette L, et al. ECR-008 study group. Inflammatory parameters associated with systemic reactogenicity following vaccination with adjuvanted hepatitis B vaccines in humans. Vaccine (2019) 37:2004-15. doi: 10.1016/j.vaccine.2019.02.015

3. Weiner J, Lewis DJM, Maertzdorf J, Mollenkopf HJ, Bodinham C, Pizzoferro $\mathrm{K}$, et al. Characterization of potential biomarkers of reactogenicity of licensed antiviral vaccines: randomized controlled clinical trials conducted by the BIOVACSAFE consortium. Sci Rep (2019) 9:20362. doi: 10.1038/ s41598-019-56994-8

4. Paine NJ, Ring C, Bosch JA, Drayson MT, Veldhuijzen van Zanten JJ. The time course of the inflammatory response to the Salmonella typhi vaccination. Brain Behav Immun (2013) 30:73-9. doi: 10.1016/ j.bbi.2013.01.004

5. Steinman RM, Hemmi H. Dendritic cells: translating innate to adaptive immunity. Curr Top Microbiol Immunol (2006) 311:17-58. doi: 10.1007/3540-32636-7_2

6. Behrens EM, Koretzky A. Review: Cytokine Storm Syndrome: Looking Toward the Precision Medicine Era. Arthritis Rheumatol (2017) 69:1135-43. doi: 10.1002/art.40071

7. Ye Q, Wang B, Mao J. The pathogenesis and treatment of the 'Cytokine Storm' in COVID-19. J Infect (2020) 80:607-13. doi: 10.1016/ j.jinf.2020.03.037

8. Shimabukuro-Vornhagen A, Gödel P, Subklewe M, Stemmler HJ, Schlößer HA, Schlaak M, et al. Cytokine release syndrome. J Immunother Cancer (2018) 6:56. doi: 10.1186/s40425-018-0343-9

9. Verschoor CP, Lelic A, Parsons R, Evelegh C, Bramson JL, Johnstone J, et al. Serum C-Reactive Protein and Congestive Heart Failure as Significant Predictors of Herpes Zoster Vaccine Response in Elderly Nursing Home Residents. J Infect Dis (2017) 216:191-7. doi: 10.1093/infdis/jix257

10. Frasca D, Blomberg BB. Inflammaging decreases adaptive and innate immune responses in mice and humans. Biogerontology (2016) 17:7-19. doi: 10.1007/s10522-015-9578-8

11. Vasto S, Candore G, Balistreri CR, Caruso M, Colonna-Romano G, Grimaldi MP, et al. Inflammatory networks in ageing, age-related diseases and longevity, Mech. Ageing Dev (2007) 128:83-91. doi: 10.1016/ j.mad.2006.11.015

12. Elias-Smale SE, Kardys I, Oudkerk M, Hofman A, Witteman JC. C-reactive protein is related to extent and progression of coronary and extra-coronary atherosclerosis; results from the Rotterdam study. Atherosclerosis (2007) 195: e195e202. doi: 10.1016/j.atherosclerosis.2007.07.006

13. Hussain SP, Harris CC. Inflammation and cancer: an ancient link with novel potentials. Int J Cancer (2007) 121:2373-80. doi: 10.1002/ijc.23173

14. Grivennikov SI, Greten FR, Karin M. Immunity, inflammation, and cancer. Cell (2010) 140:883-99. doi: 10.1016/j.cell.2010.01.025

15. Pelosof LC, Gerber DE. Paraneoplastic syndromes: an approach to diagnosis and treatment. Mayo Clin Proc (2010) 85:838-54. doi: 10.4065/ mcp. 2010.0099

16. Illman J, Corringham R, Robinson DJr, Davis HM, Rossi JF, Cella D, et al. Are inflammatory cytokines the common link between cancer-associated cachexia and depression? J Support Oncol (2005) 3:37-50.

17. Delano MJ, Ward PA. The immune system's role in sepsis progression, resolution, and long-term outcome. Immunol Rev (2016) 274:330-53. doi: 10.1111/imr.12499

18. Dedera DA, Urashima M, Chauhan D, LeBrun DP, Bronson RT, Anderson $\mathrm{KC}$. Interleukin-6 is required for pristane-induced plasma cell hyperplasia in mice. Br J Haematol (1996) 94:53-61. doi: 10.1046/j.1365-2141.1996. 6282074

19. Gadó K, Silva S, Pálóczi K, Domján G, Falus A. Mouse plasmacytoma: an experimental model of human multiple myeloma. Haematologica (2001) 86:227-36.

20. Klein B, Bataille R. Cytokine network in human multiple myeloma. Hematol Oncol Clin North Am (1992) 6:273-84.
21. Rossi JF, Lu ZY, Jourdan M, Klein B. Interleukin-6 as a therapeutic target. Clin Cancer Res (2015) 15:1248-57. doi: 10.1158/1078-0432.CCR-14-2291

22. Rossi JF, Céballos P, Lu ZY. Immune precision medicine for cancer: a novel insight based on the efficiency of immune effector cells. Cancer Commun (Lond) (2019) 39(1):34. doi: 10.1186/s40880-019-0379-3

23. Herbel C, Patsoukis N, Bardhan K, Seth P, Weaver JD, Boussiotis VA. Clinical significance of $\mathrm{T}$ cell metabolic reprogramming in cancer. Clin Transl Med (2016) 5(1):29. doi: 10.1186/s40169-016-0110-9

24. Tcyganov E, Mastio J, Chen E, Gabrilovich DI. Plasticity of myeloid-derived suppressor cells in cancer. Curr Opin Immunol (2018) 51:76-82. doi: 10.1016/j.coi.2018.03.009

25. Sica A, Strauss L, Consonni FM, Travelli C, Genazzani A, Porta C. Metabolic regulation of suppressive myeloid cells in cancer. Cytokine Growth Factor Rev (2017) 35:27-35. doi: 10.1016/j.cytogfr.2017.05.002

26. Shimizu K, Iyoda T, Okada M, Yamasaki S, Fujii SI. Immune suppression and reversal of the suppressive tumor microenvironment. Int Immunol (2018) 30:445-54. doi: 10.1093/intimm/dxy042

27. Guthrie GJ, Charles KA, Roxburgh CS, Horgan PG, McMillan DC, Clarke SJ. The systemic inflammation-based neutrophil-lymphocyte ratio: experience in patients with cancer. Crit Rev Oncol Hematol (2013) 88:218-30. doi: 10.1016/j.critrevonc.2013.03.010

28. Nistico P, Ciliberto G. Biological mechanisms linked to inflammation in cancer: discovery of tumor microenvironment-related biomarkers and their clinical application in solid tumors. Int J Biol Markers (2020) 35(S1):8-11. doi: 10.1177/1724600820906155

29. Engin A. The Pathogenesis of Obesity-Associated Adipose Tissue Inflammation. Adv Exp Med Biol (2017) 960:221-45. doi: 10.1007/978-3319-48382-5_9

30. Medzhitov R. Origin and physiological roles of inflammation. Nature (2008) 454:428-35. doi: 10.1038/nature07201

31. Yang D, Han Z, Oppenheim JJ. Alarmins and immunity. Immunol Rev (2017) 280:41-56. doi: 10.1111/imr.12577

32. Amarante-Mendes GP, Adjemian S, Branco LM, Zanetti LC, Weinlich R, Bortoluci KR. Pattern recognition receptors and the host cell death molecular machinery. Front Immunol (2018) 9:2379. doi: 10.3389/ fimmu.2018.02379

33. Grazioli S, Pugin J. Mitochondrial damage-associated molecular patterns: from inflammatory signaling to human disease. Front Immunol (2018) 9:832. doi: 10.3389/fimmu.2018.00832

34. Malik A, Kanneganti TD. Inflammasome activation and assembly at a glance. J Cell Sci (2017) 130:3955-63. doi: 10.1242/jcs.207365

35. Fraser DA, Tenner AJ. Directing an appropriate immune response: the role of defense collagens and other soluble pattern recognition molecules. Curr Drug Targ (2008) 9:113-22. doi: 10.2174/138945008783502476

36. Vanaja SK, Rathinam VA, Fitzgerald KA. Mechanisms of inflammasome activation: recent advances and novel insights. Trends Cell Biol (2015) 25:308-15. doi: 10.1016/j.tcb.2014.12.009

37. Rathinam VAK, Fitzgerald KA. Inflammasome Complexes: Emerging Mechanisms and Effector Functions. Cell (2016) 5:792-800. doi: 10.1016/ j.cell.2016.03.046

38. Kolaczkowska E, Kubes P. Neutrophil recruitment and function in health and inflammation. Nat Rev Immunol (2013) 13:159-75. doi: 10.1038/nri3399

39. Mortaz E, Alipoor SD, Adcock IM, Mumby S, Koenderman L. Update on Neutrophil Function in Severe Inflammation. Front Immunol (2018) 2 (9):2171. doi: 10.3389/fimmu.2018.02171

40. Griffiths HR, Dias IH, Willetts RS, Devitt A. Redox regulation of protein damage in plasma. Redox Biol (2014) 2:430-5. doi: 10.1016/ j.redox.2014.01.010

41. Pittman K, Kubes P. Damage-associated molecular patterns control neutrophil recruitment. J Innate Immuno (2013) 5:315-23. doi: 10.1159/ 000347132

42. Castanheira FVS, Kubes P. Neutrophils and NETs in modulating acute and chronic inflammation. Blood (2019) 133:2178-85. doi: 10.1182/blood-201811-844530

43. Brinkmann V, Reichard U, Goosmann C, Fauler B, Uhlemann Y, Weiss DS, et al. Neutrophil extracellular traps kill bacteria. Science (2004) 303:1532-5. doi: $10.1126 /$ science. 1092385 
44. Yousefi S, Mihalache C, Kozlowski E, Schmid I, Simon HU. Viable neutrophils release mitochondrial DNA to form neutrophil extracellular traps. Cell Death Differ (2009) 16:1438-44. doi: 10.1038/cdd.2009.96

45. Welin A, Amirbeagi F, Christenson K, Björkman L, Björnsdottir H, Forsman $\mathrm{H}$, et al. The human neutrophil subsets defined by the presence or absence of OLFM4 both transmigrate into tissue in vivo and give rise to distinct NETs in vitro. PLoS One (2013) 8(7):e69575. doi: 10.1371/journal.pone.0069575

46. Alder MN, Opoka AM, Lahni P, Hildeman DA, Wong HR. Olfactomedin-4 is a candidate marker for a pathogenic neutrophil subset in septic shock. Crit Care Med (2017) 45:e426-32. doi: 10.1097/CCM.0000000000002102

47. Hesselink L, Spijkerman R, van Wessem JP, Koenderman L, Leenen LPH, Huber-Lang M, et al. Neutrophil heterogeneity and its role in infectious complications after severe trauma. W Emerg Surg (2019) 14:24. doi: 10.1186/ s13017-019-0244-3

48. Khan AA, Alsahli MA, Rahmani AH. Myeloperoxidase as an active disease biomarker: recent biochemical and pathological perspectives. Med Sci (2018) 6:33. doi: $10.3390 /$ medsci6020033

49. Kumar KP, Nicholls AJ, Wong CHY. Partners in crime: neutrophils and monocytes/macrophages in inflammation and disease. Cell Tissue Res (2018) 371:551-65. doi: 10.1007/s00441-017-2753-2

50. Shi C, Pamer EG. Monocyte recruitment during infection and inflammation. Nat Rev Immunol (2011) 11:762-74. doi: 10.1038/nri3070

51. Ginhoux F, Jung S. Monocytes and macrophages: developmental pathways and tissue homeostasis. Nat Rev Immunol (2014) 14:392-404. doi: 10.1038/ nri3671

52. Takano T, Azuma N, Satoh M, Toda A, Hashida Y, Satoh R, et al. Neutrophil survival factors (TNF-alpha, GM-CSF, and G-CSF) produced by macrophages in cats infected with feline infectious peritonitis virus contribute to the pathogenesis of granulomatous lesions. Arch Virol (2009) 154:775-81. doi: 10.1007/s00705-009-0371-3

53. Hamilton TA, Zhao C, Pavicic PG, Datta S. Myeloid Colony-stimulating factors as regulators of macrophage polarization. Front Immunol (2014) 5:554. doi: 10.3389/fimmu.2014.00554

54. Liu C, Li Y, Yu J, Feng L, Hou S, Liu Y, et al. Targeting the shift from M1 to M2 macrophages in experimental autoimmune encephalomyelitis mice treated with Fasudil. PLoS One (2013) 8:e54841. doi: 10.1371/ journal.pone.0054841

55. Faix JD. Biomarkers of sepsis. Crit Rev Lab Sci (2013) 50:23-36. doi: 10.3109/ 10408363.2013.764490

56. Barma M, Goodbrand JA, Donnan PT, Mc Gilchrist MM, Frost H, McMurdo MET, et al. Slower decline in C-reactive protein after an inflammatory insult is associated with longer survival in older hospitalized patients. PLos One (2016) 11(7):e0159412. doi: 10.1371/journal.pone. 0159412

57. Monneret G, Lepape A, Voirin N, Bohe J, Vehet F, Debard A-L, et al. Persisting low monocyte human leukocyte antigen-DR expression predicts mortality in septic shock. Intensive Care Med (2006) 32:1175-83. doi: 10.1007/s00134-006-0204-8

58. Silvin A, Chapuis N, Dunsmore G, Goubet AG, Dubuisson A, Derosa L, et al. Elevated calprotectin and abnormal myeloid cell subsets discriminate severe from mild COVID-19. Cell (2020) 182:1401-8.e18. doi: 10.1016/ j.cell.2020.08.002

59. Ahmed KA, Munegowda MA, Xie Y, Xiang J. Intercellular trogocytosis plays an important role in modulation of immune responses. Cell Mol Immunol (2008) 5:261-9. doi: 10.1038/cmi.2008.32

60. Rosenits K, Keppler SJ, Vucikuja S, Aichele P. T cells acquire cell surface determinants of APC via in vivo trogocytosis during viral infections. Eur J Immunol (2010) 40:3450-7. doi: 10.1002/eji.201040743

61. Seidelin JB, Nielsen OH, Strøm J. Soluble L-selectin levels predict survival in sepsis. Intensive Care Med (2002) 28:1613-8. doi: 10.1007/s00134-002-15011505

62. Zou Q, Wen W, Zhang XC. Presepsin as a novel sepsis biomarker. World J Emerg Med (2014) 5:6-19. doi: 10.5847/wjem.j.issn.1920-8642.2014.01.002

63. Hirano T, Yasukawa K, Harada H, Taga T, Watanabe Y. Matsuda, TComplementary DNA for a novel human interleukin (BSF-2) that induces B lymphocytes to produce immunoglobulin. Al Nature (1986) 324:73-6. doi: $10.1038 / 324073 \mathrm{a} 0$
64. Yang Y, Shen C, Li J, Yuan J, Wei J, Huang F, et al. Plasma IP-10 and MCP-3 levels are highly associated with disease severity and predict the progression of COVID-19. J Allergy Clin Immunol (2020) 146:119-127.e4. doi: 10.1016/ j.jaci.2020.04.027

65. Liao M, Liu Y, Yuan J, Wen Y, Xu G, Zhao J, et al. Single-cell landscape of bronchoalveolar immune cells in patients with COVID-19. Nat Med (2020) 26:842-4. doi: 10.1038/s41591-020-0901-9

66. Saraiva M, O'Garra A. The regulation of IL-10 production by immune cells. Nat Rev Immunol (2010) 10:170-81. doi: 10.1038/nri2711

67. Redford PS, Murray PJ, O'Garra A. The role of IL-10 in immune regulation during M. Tuberculosis Infect Mucosal Immunol (2011) 4:261-70. doi: $10.1038 / \mathrm{mi} .2011 .7$

68. Mannino MH, Zhu Z, Xiao H, Bai Q, Wakefield MR, Fang Y. The paradoxical role of IL-10 in immunity and cancer. Cancer Lett (2015) 367:103-7. doi: 10.1016/j.canlet.2015.07.009

69. Ouyang W, O'Garra A. Family cytokines IL-10 and IL-22: from basic science to clinical translation. Immunity (2019) 50:871-91. doi: 10.1016/ j.immuni.2019.03.020

70. Kishimoto T. Interleukin-6: from basic science to medicine-40 years in immunology. Annu Rev Immunol (2005) 23:1-21. doi: 10.1146/ annurev.immunol.23.021704.115806

71. Heinrich PC, Castell JV, Andus T. Interleukin-6 and the acute phase response. Biochem J (1990) 265:621-36. doi: 10.1042/bj2650621

72. Nemeth E, Rivera S, Gabayan V, Keller C, Taudorf S, Pedersen BK, et al. IL-6 mediates hypoferremia of inflammation by inducing the synthesis of the iron regulatory hormone hepcidin. J Clin Invest (2004) 113:1271-6. doi: 10.1172/ JCI20945

73. Schultz DR, Arnold PI. Properties of four acute phase proteins: C-reactive protein, serum amyloid A protein, alpha 1-acid glycoprotein, and fibrinogen. Semin Arthritis Rheumatol (1990) 20:129-47. doi: 10.1016/0049-0172(90) 90055-k

74. Volanakis JE. Human C-reactive protein: expression, structure and function. Mol Immunol (2001) 38:189-97. doi: 10.1016/s0161-5890(01)00042-6

75. Ganapathi MK, Rzewnicki D, Samols D, Jiang SL, Kushner I. Effect of combinations of cytokines and hormones on synthesis of serum amyloid A and C-reactive protein in Hep 3B cells. J Immunol (1991) 147:1261-5.

76. Guo YZ, Pan L, Du CJ, Ren DQ, Xie XM. Association between C-reactive protein and risk of cancer: a meta-analysis of prospective cohort studies, Asian Pac. J Cancer Prev (2013) 14:243-8. doi: 10.7314/apjcp.2013.14.1.243

77. Wang Y, Zhang Z, Wang J, Zhang X. Association between C-reactive protein level and subsequent risk of ovarian cancer. Medicine (2020) 90:e18821. doi: 10.1097/MD.0000000000018821

78. Garcia-Anguita A, Kakourou A, Tsilidis KK. Biomarkers of inflammation and immune function and risk of colorectal cancer. Curr Colorectal Cancer Rep (2015) 11:250-8. doi: 10.1007/s11888-015-0282-5

79. Li Y, Zhong X, Cheng G, Zhao C, Zhang L, Hong H, et al. Hs CRP and allcause, cardiovascular, and cancer mortality risk: a meta-analysis. Atheroscler (2017) 259:75-82. doi: 10.1016/j.atherosclerosis.2017.02.003

80. Allin KH, Nordestgaard BG. Elevated C-reactive protein in the diagnosis, prognosis, and cause of cancer. Crit Rev Clin Lab Sci (2011) 48:155-70. doi: $10.3109 / 10408363.2011 .599831$

81. Zhang Y, Jiang L. CRP $1059 \mathrm{G} / \mathrm{C}$ and 1846G/A polymorphisms and cancer risk: a meta-analysis of 26,634 subjects. Clin Res Hepatol Gastroenterol (2014) 38:607-12. doi: 10.1016/j.clinre.2014.04.002

82. Siemes C, Visser LE, Coebergh JW, Splinter TA, Witteman JC, Uitterlinden $\mathrm{AG}$, et al. C-reactive protein levels, variation in the C-reactive protein gene, and cancer risk: the Rotterdam Study. J Clin Oncol (2006) 24:5216-22. doi: 10.1200/JCO.2006.07.1381

83. Eggimann P, Que YA, Rebeaud F. Measurement of pancreatic stone protein in the identification and management of sepsis. Biomark Med (2019) 13:135-45. doi: 10.2217/bmm-2018-0194

84. Aloisio E, Dolci A, Panteghini M. Procalcitonin: between evidence and critical issues. Clin Chim Acta (2019) 496:7-12. doi: 10.1016/ j.cca.2019.06.010

85. Barassi A, Pallotti F, Melzi d'Eril GV. Biological variation of procalcitonin in healthy individuals, Clin. Chem (2004) 50:1878. doi: 10.1373/ clinchem.2004.037275 
86. Karakioulaki M, Stolz D. Biomarkers in pneumonia. Beyond procalcitonin. Int J Med Sci (2019) 20:2-18. doi: 10.3390/ijms20082004

87. Salluh JIF, Souza-Dantas VC, Povoa P. The current status of biomarkers for the diagnosis of nosocomial pneumonias. Curr Opin Crit Care (2017) 23:391-7. doi: 10.1097/MCC.0000000000000442

88. Coomes EA, Hayghbayan H. Interleukin-6 in COVID-19: a systematic review and meta-analysis. Rev Med Virol (2020) 30:1-9. doi: 10.1002/rmv.2141

89. Luo P, Liu Y, Qiu L, Liu X, Ji L. Tocilizumab treatment in COVID-19: a single center experience. J Med Virol (2020) 92:814-8. doi: 10.1002/jmv.25801

90. Zhang Q, Prabhu A, San A, Al-Sharab JF, Levon K. A polyaniline based ultrasensitive potentiometric immunosensor for cardiac troponin complex detection. Biosens Bioelectron (2015) 72:100-6. doi: 10.1016/ j.bios.2015.04.084

91. Ridker PM. Anticytokine agents: targeting interleukin signaling pathways for the treatment of atherothrombosis. Circ Res (2019) 124:437-50. doi: 10.1161/CIRCRESAHA.118.313129

92. Alexia C, Cren M, Louis-Plence P, Vo DN, El Ahmadi Y, Dufourcq-Lopez E, et al. Polyoxidonium ${ }^{\circledR}$ activates cytotoxic lymphocyte response through dendritic cell maturation: clinical effects in breast cancer. Front Immunol (2019) 10:2693. doi: 10.3389/fimmu.2019.02693

93. Frick JS, Grünebach F, Autenrieth IB. Immunomodulation by semi-mature dendritic cells: a novel role of Toll-like receptors and interleukin-6. Int J Med Microbiol (2010) 300:19-24. doi: 10.1016/j.ijmm.2009.08.010

94. Bouras M, Asehnoune K, Roquilly A. Contribution of dendritic cell responses to sepsis-induced immunosuppression and to susceptibility to secondary pneumonia. Front Immunol (2018) 9:2590. doi: 10.3389/ fimmu.2018.02590

95. Correale P, Botta C, Staropoli N, Nardone V, Pastina P, Ulivieri C, et al. Systemic inflammatory status predict the outcome of k-RAS WT metastatic colorectal cancer patients receiving the thymidylate synthase poly-epitopepeptide anticancer vaccine. Oncotarget (2018) 9:20539-54. doi: 10.18632/ oncotarget. 24993

96. Laino AS, Woods D, Vassallo M, Qian X, Tang H, Wind-Rotolo M, et al. Serum interleukin-6 and C-reactive protein are associated with survival in melanoma patients receiving immune checkpoint inhibition. Immunother Cancer (2020) 8(1):e000842. doi: 10.1136/jitc-2020-000842

97. Damuzzo V, Solito S, Pinton L, Carrozzo E, Valpione S, Pigozzo J, et al. Clinical implication of tumor-associated and immunological parameters in melanoma patients treated with ipilimumab. Oncoimmunol (2016) 5(12): e1249559. doi: 10.1080/2162402X.2016.1249559
98. Kitamura H, Ohno Y, Toyoshima Y, Ohtake J, Homma S, Kawamura H, et al. Interleukin-6/STAT3 signaling as a promising target to improve the efficacy of cancer immunotherapy. Cancer Sci (2017) 108:1947-52. doi: $10.1111 /$ cas. 13332

99. Del Giudice M, Gangestad SW. Rethinking IL-6 and CRP: why they are more than inflammatory biomarkers, and why it matters. Brain Behav Immun (2018) 70:61-75. doi: 10.1016/j.bbi.2018.02.013

100. McFadyen JD, Kiefer J, Braig D, Loseff-Silver J, Potempa LA, Eisenhardt SU, et al. Dissociation of c-reactive protein localizes and amplifies inflammation: evidence for a direct biological role of r-reactive protein and Its conformational changes. Front Immunol (2018) 9:1351. doi: 10.3389/ fimmu.2018.01351

101. Pampena MB, Cartar HC, Cueto GR, Levy EM, Blanco PA, Barrio MM, et al. Dissecting the immune stimulation promoted by CSF- 470 vaccine plus adjuvants in cutaneous melanoma patients: long term antitumor immunity and short term release of acute inflammatory reactants. Front Immunol (2018) 9:2531. doi: 10.3389/fimmu.2018.02531

102. Sarnaik AA, Yu B, Yu D, Morelli D, Hall M, Bogle D, et al. Extended dose ipilimumab with a peptide vaccine: immune correlates associated with clinical benefit in patients with resected high-risk stage IIIc/IV melanoma. Clin Cancer Res (2011) 17:896-906. doi: 10.1158/1078-0432. CCR-10-2463

103. Ribas A, Kefford R, Marshall MA, Punt CJ, Haanen JB, Marmol M, et al. Evaluation of baseline serum C-reactive protein (CRP) and benefit from tremelimumab compared to chemotherapy in first-line melanoma. J Clin Oncol (2010) 31(5):616-22. doi: 10.1200/JCO.2012.44.6112

Conflict of Interest: J-FR, KL, and CM are co-founders of E-Sana. J-FR is consultant for Leo Pharma, NPO Petrovax and Eusapharma.

The remaining author declares that the research was conducted in the absence of any commercial or financial relationships that could be construed as a potential conflict of interest.

Copyright (c) 2021 Rossi, Lu, Massart and Levon. This is an open-access article distributed under the terms of the Creative Commons Attribution License (CC BY). The use, distribution or reproduction in other forums is permitted, provided the original author(s) and the copyright owner(s) are credited and that the original publication in this journal is cited, in accordance with accepted academic practice. No use, distribution or reproduction is permitted which does not comply with these terms. 